\title{
La Sub-Capitalización de Activos Intelectuales en América Latina
}

\author{
José Fernández Donoso*
}

\section{Prefacio}

Hoy en día, los activos intangibles como la propiedad intelectual (PI) constituyen el epicentro del valor empresarial de las multinacionales. Desafortunadamente, América Latina se encuentra rezagada y necesita de una gran revolución en sus políticas de innovación orientada a desarrollar mercados de tecnologías.

Si los líderes de empresas latinoamericanas desean innovar, deberán superar ciertos procesos cognitivos que limitan el uso de estrategias de PI. En este sentido, los gobiernos pueden hacer mucho para superar dichas barreras.

\section{Introducción}

Actualmente, "la propiedad intelectual es la moneda de la economía del conocimiento" (Ghafele \& Gibert 2012). El conocimiento de estrategias de propiedad intelectual no debiera ser encajonado como dominio exclusivo de las oficinas de transferencia tecnológica (como ocurre hoy en la región). De seguir así, las empresas innovadoras corren el riesgo de no rentabilizar sus esfuerzos creativos e innovadores por desconocer las estrategias óptimas de apropiación y comercialización de sus ideas. Además, la incapacidad para rentabilizar activos intelectuales aumenta los riegos de inversión en nuevas empresas, lo que impacta directamente en sus costos de financiamiento (De León \& Fernandez Donoso 2016a).

Existen distintas estrategias para gestionar apropiadamente los activos intelectuales. La estrategia óptima para cada activo no necesariamente requiere el uso de derechos de propiedad intelectual. Por ejemplo, se puede proteger una innovación aumentando la complejidad del diseño de componentes que dificulten su ingeniería inversa (Hall et al. 2014), separando procesos de estratégicamente para dificultar que sean replicados (Keupp et al. 2009) o simplemente porque la complejidad tecnológica es en sí una barrera para imitar un producto o proceso (Fernandez Donoso 2014) o porque el ciclo de vida producto es lo suficientemente corto como para hacer inviable la absorción y réplica del conocimiento (Bilir 2014).

A continuación, analizaré algunos de los limitantes para el uso de estas estrategias y para el desarrollo de mercados de tecnología en la región

\section{Estrategias de Propiedad Intelectual}

La solución no es simple dado que la comercialización exitosa de activos intangibles es, en sí misma, un puzle complejo. En 2015, apenas el 9\% de las PYMEs europeas eran propietarias de algún derecho de propiedad intelectual. En promedio, éstas generaban un ingreso 32\% mayor por empleado (OHIM 2015) que sus pares sin activos intelectuales. Por otro lado, de los 2,1 millones de patentes activas en Estados Unidos en 2014, el 95\% no pudieron ser comercializadas exitosamente - entre ellas 50.000 patentes de invención universitarias de alta calidad (Walker 2014) - . Por su lado, América Latina apenas representa el 2,5\% de las patentes mundiales (WIPO 2014). Del total de patentes latinoamericanas, sólo un $12 \%$ son solicitadas por empresas residentes (WIPO 2014) y todos los países de la región, sin excepción, muestran ingresos netos negativos por licenciamiento ${ }^{2}$.

Entre otros inconvenientes, la teoría de propiedad intelectual convencional (Machlup 1958, Kitch 1977, Besen \& Raskind 1991, Bessen 2005) no analiza la gestión de propiedad intelectual, ni por qué las empresas eligen determinadas estrategias de propiedad intelectual. En el libro "Innovation, Startups and Intellectual Property Management" (De León \& Fernández Donoso 2017), se identifican cuatro grandes categorías de estrategias de gestión de la propiedad intelectual:

(i) Estrategias Convencionales: son aquellas en las que se ejerce poder de mercado a través de la propiedad intelectual. Incluyen la diversificación de portafolios a través de distintas formas de propiedad intelectual, el desarrollo de tecnologías alternativas para competir con variedades, la publicación defensiva para limitar la competencia, la "donación" de patentes o las cláusulas de no competencia.

(ii) Estrategias colaborativas de propiedad intelectual: definen habilidades que pueden ser desarrolladas con otros negocios y cómo los títulos de propiedad intelectual pueden ser utilizados con otras empresas para obtener capacidades faltantes, ya sea por intercambios o por licenciamiento conocido como "patent pooling".

* Facultad de Economía y Negocios, Universidad del Desarrollo. Av. Plaza 680, Las Condes, Santiago (Chile). E-mail: jgfernandez@udd.cl (2) De acuerdo a Indicadores de Desarrollo Mundial (2015), los ingresos netos por licenciamiento de patentes (en millones de dólares de EEUU corrientes): Argentina (-1.799,9), Brasil (-5.547,6), Chile (-1.461,9), Colombia (-442,2), Costa Rica (-134), México (-368,3), Perú (-253,5), en contraste con Estados Unidos (88.237). 
(iii) Estrategias ofensivas: estrategias basadas en la litigación como activo de inversión, donde las ganancias del litigio, los costos y los riesgos son medidos con valor esperado positivo.

(iv) Estrategias de ocultación: estrategias de propiedad intelectual cuyo objetivo es no haber público un conocimiento determinado. Incluyen el uso de múltiples patentes con información segmentada, las cláusulas de confidencialidad, etc.

Al igual que en la definición de teoría de juegos, la estrategia óptima para una determinada innovación debería contemplar todas las contingencias posibles. Una estrategia puede también ser una composición de elementos pertenecientes a distintas categorías: publicación sólo de una parte de la innovación (estrategia de tipo convencional) -aunque insuficiente para ser replicada por terceras partes- y además cláusulas de confidencialidad (estrategia de ocultación).

La conveniencia de cada estrategia dependerá de diversos factores: (i) condiciones externas a la empresa - por ejemplo: industrias de alta complejidad tecnológica, absorción tecnológica de países donde se localiza la producción, protección institucional de la propiedad intelectual, etc.-, (ii) características de la innovación (Hall et al. 2014, Fernandez Donoso 2014) - por ejemplo: patentabilidad, complejidad tecnológica, ciclo del producto, diferencias inventivas con productos similares, etc. - y (iii) condiciones internas de la empresa - por ejemplo: aversión al riesgo, cultura de interna de innovación, disonancia cognitiva a sistemas formales de propiedad intelectual, entre otros.

\section{Pequeñas empresas innovadoras en América Latina y limitaciones cognitivas}

La identificación de la estrategia óptima de propiedad intelectual de cada innovación empresarial debe formar parte de la estrategia global de la empresa. Este componente -el de propiedad intelectual- es aún más importante en la estrategia empresarial de emprendimientos dinámicos, cuyo motor de crecimiento es la comercialización de activos intelectuales ${ }^{3}$. La no elaboración de una estrategia adecuada limita la capacidad para capitalizar estos activos intelectuales y, cuando la falta de estrategias es generalizada, la sub-capitalización de activos incide en el riesgo de invertir en startups.

En América Latina, este riesgo incremental está detrás de la poca disponibilidad de financiamiento privado al emprendimiento. Sin embargo, las pequeñas empresas innovadoras en América Latina muy rara vez elaboran una estrategia para proteger sus activos intelectuales (De Leon \& Fernandez Donoso 2016b). Se pueden identificar los siguientes procesos que limitan el uso de los sistemas de propiedad intelectual:
- Disonancia cognitiva: cuando hay fuertes convicciones en contra del uso de los sistemas de propiedad intelectual ("fumar es malo, quiero a fumar"). Existen empresas que, aun sabiendo la importancia de proteger y capitalizar estos activos, tienen creencias y convicciones en contra, por lo que optan por no usarlas.

- Sobrecarga cognitiva: emprender, innovar y poner en marcha un negocio requiere importantes esfuerzos cognitivos (especificidades técnicas de producto o proceso, marketing, comercial, tributación, etc.). El emprendedor (empresario) simplemente se ve desbordado en ocupaciones, dejando de lado la elaboración de una estrategia de propiedad intelectual.

- “Sheer ignorance” (Kirzner 1997): no saber cómo acceder a un conocimiento específico por el simple hecho de no saber que no se sabe.

El sesgo cognitivo detrás de estas limitaciones es un sesgo confirmatorio (De Leon \& Fernandez Donoso 2017). Entrevistando en profundidad a más de 350 empresas innovadoras de la región, buscamos cuantificar los costos efectivos de uso de los sistemas de propiedad intelectual, a partir de cuatro hipótesis iniciales: (i) costos legales excesivos, (ii) imitación una vez publicada la innovación por grandes empresas con capacidad de financiar grandes litigios, (iii) tramitación larga y costosa antes de obtener el derecho de propiedad intelectual y (iv) poca protección durante proceso de revisión del derecho de propiedad intelectual.

Adicional a una baja presencia de las hipótesis iniciales como limitantes del uso de estos derechos, identificamos la tendencia a favorecer, buscar, interpretar y recordar información que confirma la creencia de la inutilidad de los sistemas de propiedad intelectual. La desproporcionada falta de consideración a los beneficios de capitalizar activos intelectuales es un serio impedimento para el desarrollo de mercados tecnológicos y de innovación en la región.

\section{Discusión e implicancias de política}

El diseño de una estrategia pública para fomentar el uso inteligente de la propiedad intelectual requiere un previo entendimiento del ecosistema de innovación que acompaña la implementación de la estrategia. Este contexto debe tomar en cuenta factores tales como la madurez de la tecnología, las expectativas de los receptores de algún tipo de política y el compromiso de esos receptores. Existen factores a nivel macro y micro que influyen en la transferencia tecnológica. Los factores a nivel macro se determinan a nivel nacional y son más difíciles de controlar en el corto plazo (apertura comercial, disponibilidad de insumos específicos, infraestructura, institucionalidad, condiciones de mercado, etc.). Los factores a nivel micro incluyen la cultura organizacional, ética, capital social, distancia al poder, proactividad para buscar nuevas tecnologías, capacidad de absorción tecnológica y factores sociales.

(3) Un caso emblemático y popular de la región es la empresa Crystal Lagoons. Sin embargo existen otros casos de empresas en América Latina que han crecido en torno a la capitalización de activos intelectuales (ver De León \& Fernández Donoso 2017). 
El análisis cognitivo de los demandantes de derechos de propiedad intelectual es fundamental para el diseño de políticas microeconómicas que favorezcan la transferencia y comercialización de activos intelectuales. A modo de ejemplo, en Colombia se intentaron diversas reducciones a las tasas de registro de patentes sin impacto alguno en el número de patentes solicitadas (De León \& Fernández Donoso 2015). El escaso impacto de este tipo de políticas se explica sucintamente al entender las limitaciones cognitivas de los usuarios de los sistemas de propiedad intelectual.

Por otro lado, la literatura sugiere que en la región se debiesen favorecer políticas orientadas a la transferencia de capacidades, al establecimiento de culturas de protección o a la divulgación de conocimiento práctico en torno al tema de la apropiación de activos intangibles y su rentabilidad para el negocio. En cualquier caso, el puzle de la comercialización de activos intelectuales no tiene una única solución, pues la complejidad de cada nueva creación, idea de negocio, producto, algoritmo, obra literaria o fórmula para mejorar un proceso es tan único como la originalidad de sus autores.

\section{Referencias}

Besen, Stanley M., and Leo J. Raskind. "An introduction to the law and economics of intellectual property." The Journal of Economic Perspectives 5.1 (1991): 3-27.

Bessen, James. "Patents and the diffusion of technical information." Economics Letters 86.1 (2005): 121-128.

Bilir, L. Kamran. "Patent laws, product life-cycle lengths, and multinational activity." The American Economic Review 104.7 (2014): 1979-2013.

De Leon, I., and J. Fernandez Donoso. "El costo de uso de los sistemas de propiedad intelectual para pequeñas empresas innovadoras." Documentos para Discusión\# IDB-DP-373, Inter-American Development Bank (2015).

De León, Ignacio L., and Jose Fernandez Donoso. The costs of using formal intellectual property rights: a survey on small innovative enterprises in Latin America. Inter-American Development Bank, 2016a.

De León, Ignacio L., and José Fernández Donoso. "El uso de la Propiedad Intelectual en el desarrollo de los mercados de innovación." Cap. 7 "La Política de Innovación en América Latina y el Caribe". Inter-American Development Bank, 2016b.
De León, Ignacio L., and José Fernández Donoso. "Innovation, Startups and Intellectual Property Management. Strategies and Evidence from Latin America and other Regions." Springer International Publishing, 2017.

Donoso, Jose Fernandez. "Do complex inventions need less international patent protection?." Economics Letters 125.2 (2014): 278-281.

Ghafele, R. and B. Gibert. 2012. "Promoting Intellectual Property Monetization in Developing Countries." Documento de trabajo de investigación de políticas Núm. 6143. Washington, D.C.: Banco Mundial.

Hall, Bronwyn, et al. "The choice between formal and informal intellectual property: a review." Journal of Economic Literature 52.2 (2014): 375-423.

Keupp, Marcus Matthias, Angela Beckenbauer, and Oliver Gassmann. "How managers protect intellectual property rights in China using de facto strategies.” R\&d Management 39.2 (2009): 211-224.

Kirzner, I.M. (1997), Entrepreneurial Discovery and the Competitive Market Process: An Austrian Approach. Journal of Economic Literature, 35(1): 60-85.

Kitch, Edmund W. "The nature and function of the patent system." The Journal of Law and Economics 20.2 (1977): 265-290.

Machlup, Fritz. "An economic review of the patent system". No. 15. US Government Printing Office, 1958.

Office for Harmonization in the Internal Market (OHIM). 2015. "Intellectual property rights and firm performance in Europe: an economic analysis," June 2015. Disponible en: https://euipo.europa.eu/ tunnel-web/secure/webdav/guest/document_library/observatory/ documents/IPContributionStudy/phase2/executive_summary_ en.pdf

Walker, RM. 2014. "Internal and External Antecedents of Process Innovation: A review and extension”. Public Management Review, 16(1).

World Intellectual Property Organization (WIPO). 2014. "World Intellectual Property Indicators."World Intellectual Property Organization. Disponible en: http://www.wipo.int/edocs/pubdocs/en/ wipo_pub_941_2014.pdf 